confirmed increased carotid plaque formation, increased cIMT. Biologics may beneficially influence some parameters in the intracranial vessels.

Disclosure of interest None declared

\section{P095 PLASMA LEVELS OF HSP90 ARE INCREASED IN PATIENTS WITH SYSTEMIC SCLEROSIS WITH MORE SEVERE ORGAN INVOLVEMENT}

\begin{abstract}
1,2 H Storkanova*, 1,2S Oreska, ${ }^{2,3} \mathrm{M}$ Spiritovic, ${ }^{1,2} \mathrm{~K}$ Pavelka, ${ }^{1,2} \mathrm{~J}$ Vencovský, ${ }^{4} \mathrm{~J}$ Distler, 1,2 L Senolt, ${ }^{1,2} \mathrm{R}$ Becvar, ${ }^{1,2} \mathrm{M}$ Tomcik. ${ }^{1}$ Department of Rheumatology, 1st Faculty of Medicine, Charles University, Prague, Czech Republic; 'Institute of Rheumatology, Prague, Czech Republic; ${ }^{3}$ Department of Physiotherapy, Faculty of Physical Education and Sport, Charles University, Prague, Czech Republic; ${ }^{4}$ Department of Internal Medicine III, Institute for Clinical Immunology, University of Erlangen-Nuremberg, Erlangen, Germany
\end{abstract}

\subsection{6/annrheumdis-2018-EWRR2018.111}

Introduction Our previous study demonstrated that Hsp90 is overexpressed in the skin of patients with systemic sclerosis $(\mathrm{SSc})$, in cultured SSc fibroblasts and preclinical models of SSc. We showed that Hsp90 is a regulator of TGF- $\beta$ signalling and its inhibition prevents the stimulatory effects of TGF- $\beta$ on collagen synthesis and dermal fibrosis in three preclinical models of SSc. ${ }^{1}$

Objectives The aim of this study was to evaluate Hsp90 in the circulation of SSc patients and characterise its potential association with skin changes and SSc-related features.

Methods A total of 91 patients (78 females; mean age 52.7; disease duration 6.0 years; diffuse cutaneous (dc)SSc/limited cutaneous $\left.(\mathrm{lc}) S S_{c}=38 / 53\right)$ who met the ACR/EULAR 2013 criteria for SSc and 85 age-/sex- matched healthy controls (HC) were included. Plasma Hsp90 was measured by ELISA. Data are presented as median (IQR).

Results Plasma Hsp90 levels were increased in SSc patients

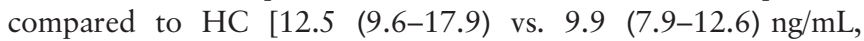
$\mathrm{p}=0.001$ ], but no difference between $\mathrm{lcSS} c$ and dcSSc were

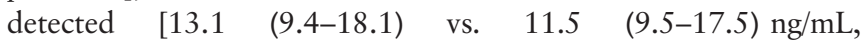
$\mathrm{p}=0.316$ ]. Hsp90 levels in all patients positively correlated with CRP $(\mathrm{r}=0.313, \mathrm{p}=0.006)$. Furthermore, Hsp90 was increased in patients with interstitial lung disease (ILD) compared to those without ILD [12.8 (10.2-17.9) vs. 10.3 (8.616.6) $\mathrm{ng} / \mathrm{mL}, \mathrm{p}=0.045]$ and was negatively associated with functional parameters of ILD: FVC $(=-0.299, \mathrm{p}=0.011)$, FEV1 $(r=-0.256, p=0.031)$, DLCO $(r=-0.303, p=0.009)$ and $\mathrm{SpO}_{2}(\mathrm{r}=-0.317, \mathrm{p}=0.038)$. In addition, only in patients with dcSSc, Hsp90 levels positively correlated with the mRSS $(r=0.437, p=0.006)$. Hsp90 concentrations were not significantly affected by other main clinical parameters of SSc.

Conclusions We demonstrated higher plasma levels of Hsp90 in SSc patients compared to healthy controls. Concentrations of extracellular Hsp90 increase with higher inflammatory activity, with deteriorated lung functions in ILD and also with the extent and severity of the skin involvement in patients with diffuse cutaneous SSc. These data further highlight the role of Hsp90 as a significant regulator of fibroblast activation and tissue fibrosis in SSc.

\section{REFERENCE}

1. Tomcik M, et al. Ann Rheum Dis 2014;73(6):1215-22.

Acknowledgements Supported by AZV - 16-33542A and SVV - 260373.

Disclosure of interest None declared

\section{P096 SYNOVIAL TISSUE REMODELLING AS A MEANS OF TISSUE MEMORY}

1,2 Olmos Calvo*, ${ }^{1} \mathrm{RA}$ Byrne, ${ }^{1} \mathrm{M}$ Bonelli, ${ }^{1} \mathrm{~B}$ Niederreiter, ${ }^{1} \mathrm{~F}$ Alasti, ${ }^{1} \mathrm{~T}$ Karonitsch, ${ }^{3} \mathrm{~J}$ Holinka, ${ }^{1} \mathrm{G}$ Steiner, ${ }^{1} \mathrm{JS}$ Smolen, ${ }^{2} \mathrm{P}$ Ertl, ${ }^{1} \mathrm{HP}$ Kiener. ${ }^{1}$ Rheumatology, Medical University of Vienna; ${ }^{2}$ Faculty of Technical Chemistry, Vienna University of Technology; ${ }^{3}$ Orthopedic Surgery, Medical University of Vienna

\subsection{6/annrheumdis-2018-EWRR2018.112}

Introduction The synovium demonstrates a distinct cellular structure with a densely packed synovial lining layer that sits on top of a loosely organised sublining layer. In rheumatoid arthritis (RA), this tissue hosts the inflammatory reaction and undergoes drastic structural changes. We hypothesise that this inflammatory remodelling results in tissue dysfunction, thereby perpetuating the inflammatory process. To explore the relationship between tissue structure and function, we use a 3D human synovial organoid culture system to model healthy and diseased synovium.

Methods FLS from RA patients were resuspended in Matrigel and cultured as 3D organoids for an extended period of time. This allows FLS to re-establish a synovial tissue-like structure. To mimic inflammation, 3D synovial organoids were challenged with TNF. A re-stimulation experiment was designed in which synovial organoids were exposed to TNF for 10 days, followed by a 3 day wash out phase. Thereafter, the organoids were re-stimulated with TNF. To prevent TNF-driven remodelling, Marimastat, a broad MMP inhibitor, was added during the first TNF stimulation. For selected experiments, synovial tissue-like structure was assessed by $\mathrm{HE}$ staining in paraffin embedded sections of synovial organoids. qPCR and RNA Seq were used in gene expression profiling. IL-6, IL-8 and MMP3 protein production was determined by ELISA.

Results TNF-stimulated synovial organoids demonstrated lining layer hyperplasia and cellular condensation in the sublining layer. After TNF-stimulation, gene expression of MMP1, MMP3 and IL-6 and protein production of IL-6, IL-8 and MMP3 was upregulated. Upon re-stimulation of synovial organoids with TNF, protein levels of IL-6, IL-8 and MMP3 were significantly increased when compared to previous stimulations. In order to explore whether this effect is due to TNFinduced structural changes, tissue remodelling was blocked using Marimastat. IL-6 protein level was reduced in 21\%. To further explore the increased TNF response upon re-stimulation, epigenetic mechanisms are currently being tested.

Conclusions TNF stimulation has a direct effetc in synovial organoids function and structure. TNF-driven tissue remodelling is associated with an increased in IL-6, IL-8 and MMP3 expression response upon re-stimulation. Inhibition of TNFinduced remodelling partially prevents this effect. Additional epigenetic mechanisms may account for tissue memory.

Disclosure of interest None declared

\section{P097 ANTI-TNF TREATMENT IMPROVES VASCULAR FUNCTION VIA SUPPRESSION OF GALECTIN-3 EXPRESSION DURING INFLAMMATORY ARTHRITIS}

K Sime*, E Hughes, EH Choy, AS Williams. Cardiff University, Cardiff, UK

\subsection{6/annrheumdis-2018-EWRR2018.113}

Introduction The link between rheumatoid arthritis (RA) and cardiovascular disease (CVD) is well established but not yet fully understood. Anti-TNF treatment, e.g. with Enbrel, is one 